\title{
International City Traffic Congestion Based Analysis and Countermeasures Research
}

\author{
Fei Li \\ Zhongshan Avenue 12 Government-Run, Xuanhua District, Zhangjiakou City, Hebei Province, China \\ E-mail: 1f5819@sina.com \\ Received January 6, 2011; revised January 19, 2011; accepted January 24, 2011
}

\begin{abstract}
New York, London, Paris and Tokyo, these world-class cities have traffic congestion problems. Paper pointed out that the main reason for traffic jams is incompatible with transport planning and transport development, the Metropolitan Transportation properly classified according to physical distance, to borrow the metaphor of Chinese classical culture, that "pie", "Gun Xiuqiu”, "sword cut" and "kite flying” and four are independent, interconnected transportation planning program. Use of an important node in the network traffic, the transportation planning program four seamless integration, expanding the concept of traffic, build traffic new venues to meet the sea, land and waited in vain for multi-functional travel needs through one-way loop that Basic theory, integrated network group, established metropolitan cellular transport network system, with sophisticated network management software, intelligent transportation and immediate traffic management methods to various means of transportation in big cities of the diversion of human nature, which break the cities Traffic congestion, to achieve smooth traffic international cities.
\end{abstract}

Keywords: Cities, Traffic Congestion, One-Way Loop, Cellular Transport Network Group

\section{Introduction}

In the world, with the rapid increase in the number of transport, transport function of the expansion of diversified transportation trends in the world. States cities have developed many ways to implement various measures, still exist to varying degrees, the phenomenon of traffic congestion. The root causes of traffic jams caused by big cities, where is? To solve this problem, the physical distance of major cities were classified, and borrowed the image of the Chinese classic culture metaphor that "pie", "Gun Xiuqiu", "cutting sword" and "kite flying" and four are independent, Layered access, interoperability of transportation planning programs, the establishment of metropolitan transport network model of honeycomb from the big city traffic aspects of hardware and software to solve traffic congestion problems large cities [1].

\section{First, Build the Hardware Platform to Solve Urban Congestion}

\section{1. "Pie" Planning [2]}

This is the traditional mode of transportation planning and construction around the world are present, people with disabilities Road, walking trails, bike paths, lanes and other traffic criss-cross the formation of a typical straight-line box pie, as city residents most frequently used daily travel Channel, is also the basis for the city channel. The program is mainly responsible for solving the short-distance traffic roaming city alley.

\section{2. “Gun Xiuqiu” Planning}

Establish a priority in urban transport planning the construction of new ideas, through one-way circulation patterns, building large cities cellular transport network group, since any two adjacent rings are the same between the driving direction, so the abolition of the traffic lights in the traffic network settings. The program is mainly responsible for address range through the city (such as multi-center, satellite) in the middle of traffic.

Cellular transport network model is the most significant features: open. In the computer network ring topology, the network data transmission characteristics of a one-way, to this principle in the map to the transport network is one-way loop road transport network in the traffic jam phenomenon does not realize the big city traf- 
fic from “A red” to "a green” changes.

\section{3. "Cutting Sword” Planning}

As the Chinese saying "one asked in the end", the proverb, illustrates the meaning of this plan, under the lines in this plan, regardless of how much each is to achieve "line in the end", the one-off transport through the city, not stay the way. As a line which is like a melon, a knife down, the "city" split in half. The program is mainly responsible for solving the long-distance traffic across the city.

\section{4. "Kite Flying” Planning}

Similar to the airport as "small but perfectly formed", but a much smaller scale than the airport, more affordable, and kite flying is similar to that in the transport network in the new transport landing site, if it is the coastal city, But also planning of transport access to the sea port. The program primarily responsible for solving the sky and the sea, and other special transportation.

Need of special note is that only four types of transport planning principles and models, is the topology, different countries to combine their own national conditions, according to the local urban population, land area, vehicle type, degree of openness such as to make the field of space Reasonable and scientific decisions.

\section{In Beijing, China, for Example, the Establishment of Beijing Transportation Network Model of Honeycomb in Order to Further Understanding and Knowledge of the System}

Assume that China's urban transportation planning for the Beijing radius $300 \times 300$ square kilometers, if the basic unit of $10 \mathrm{~km}$, then to form a total of $90030 \times 30$ cellular transport network. Transportation Planning four as follows:

$0 \mathrm{~km}$ away, in accordance with the "pie” Planning building. The formation of $(300 / 10) \times(300 / 10)$ Total 900 groups of urban construction land, the planning of new groups, a small city as cities group, which contains a huge wealth. In order to build a livable city Beijing to achieve natural and harmonious living environment, the proposed alternative construction of $10 \mathrm{~km}$ every есоlogical zone, the ecological zone as the city's natural forests, the lungs of the city.

10 kilometers, according to "Gun Xiuqiu” planning construction. The formation of $(300 / 10) \times(300 / 10)$ Total 900 network traffic lines. This is the main form of one-way loop road, but also solve traffic congestion in Beijing an important part.

20 kilometers, according to "kite flying" Planning building. The formation of $(300 / 20) \times(300 / 20)$ Total 225 "kite flying” space. According to Beijing put the number of aircraft and air traffic control requirements for construction, transport services have been set up for each site area, to respond to emergencies, the implementation of traffic control.

30 kilometers, according to "cut sword", Planning building. The formation of $(300 / 30) \times(300 / 30)$ in Beijing a total of 100 across the "bridge". It should be noted that the daily closure of the footbridge node, if you encounter special circumstances, such as the outbreak of war, node collapse, heavy rain snow and so on, around each node based on the actual need of immediate opening, so that the formation of a larger transportation network, prevention of urban Traffic was paralyzed in order to meet the disposal of urban forces, mass transfer, material supply and other emergency needs.

Through a century building, Beijing, China hopes to achieve this goal: blue sky, the capital city as red and green chessboard, shade, such as jade, Red House, such as precious stones, thread, such as gold, undulating like ocean waves, such as Heaven Habitat, driving, such as flying. Beijing, China will build into a large number of Beijing, a large forest in Beijing, a large network in Beijing, smooth big Beijing.

\section{Closely with the Four Transportation Planning Program, it is Also Important to Note the Following Questions}

\subsection{Cause the Four Focus}

\subsubsection{Open the City's Airspace}

Aircraft had been "parachuted" to the residents living in the hope that countries according to their actual situation, the city-owned open space areas, open up a section for people to air channel. Otherwise, the people have not cooked rice without rice pot. For example, Manhattan, New York in 2010 to make a "fly" to work, an active open space aviation authority to allow private plane took off [3].

\subsubsection{Facturing Airbus}

Pyramid, solar appearance, is divided into $\mathrm{N}$ layers (e.g., $\mathrm{N}=7$ ), to accommodate tens of thousands and thousands of cars, using solar energy and electricity can drive. In order to save the city's land, the main Airbus pyramid cities across the main channel in the "floating" operation, from the air, overlooking one of Airbus as "moving” of the pyramid, keep the shuttle in the city, classical and 
stylish , the existence of the pyramid, will be the largest city in a beautiful landscape. Meanwhile, in the network nodes in all important transfer station to be set up.

This is the world faces a new task. This "walking the Pyramid" has a lot of advantages: load capacity, low energy consumption, low-carbon nature, less pollution, noise, environmental protection is good, driving fast, a short distance and efficiency. Daily traffic can be "passengers sightseeing, car travel”. Engineers also want to stick to one pattern, designed to meet the future transport needs of a wide variety of transport.

\subsubsection{Universal Rail Transportation}

In the cellular transport network should cover the entire rail network of more than $80 \%$, to a high starting point plan, generous construction, all the nodes to achieve a one-stop transfer. So people can save time and improve efficiency. In large cities, construction of rail traffic is very necessary and very important, cause some countries to pay special attention, do not be taken lightly. The public will that they will choose the most economical way to determine their own travel, or even a region will affect the price of the traffic heavy traffic in the region and depression. For example, in November 2010, China "Guangzhou Daily” reported: Guangzhou Free Metro crowded, traffic is very "hot" than the previous day by more than 200 free people. Only "0" price subway on the "Day" by pulling 200 million people [4]. This message reflects the mass psychology of a trend: the majority of people still hope that public transport prices as low as possible, and even a free or subsidized travel. 200 million people in the subway, may reduce the tens of thousands of traffic to Guangzhou or hundreds of thousands of vehicles, greatly reduced the traffic pressure, understanding and knowledge of public preferences for modes of transportation, for the problems of urban congestion has a very important role, because people understand the city Preferences, you can modify the established traffic patterns and reduce the probability of congestion. The city in large cities, the number of rail traffic and transport network is much improved, can be said to be a barometer of the efficiency of urban transit and compass, is the urban traffic congestion is frequently an important factor.

\subsubsection{Pay Close Attention to the Node Construction}

Three-dimensional transport can not be separated to achieve the construction of transportation hub, an important node bears a very busy transit work, although the four transport non-interference between channels, but the traffic through the node while ensuring interoperability of four designs on the world sea Security design, etc., so the scientific design of the node is very important in solving urban traffic congestion, and plays a role in con- necting link.

\subsection{To Do Three Interfaces}

\subsubsection{Contains the Entrance}

In an important node, open up "Airbus” loading interface, play fast bulk transfer transport role. If in a particular region severe traffic jams, congestion in the road transport will set out the entrance and transferred to Airbus to go, then Airbus is responsible for the "add traffic jam" transport to the destination. Transport take a "free" air transportation. On the monitor will display part of the vehicle suddenly in the congested road "collective disappear", thus easing the traffic congestion of the road.

\subsubsection{Take off and Land Ports}

In a dynamic driving on the road, sometimes the density of vehicles increases suddenly and may jeopardize the safety of aircraft took off from, in order to avoid traffic safety problems, the network needs to establish a number of aircraft landing field, aims to have the fly Function of transport available to take off and landing entrances and exits. So to have the air-land transport amphibious released into the sky, reducing the number of road transport to ease traffic congestion in the road. In many nodes, landing field is a multi-design, an increase of urban capacity, but also ease the difficult parking problems.

Of course, in the case of smooth road, with flying capabilities of transport on the road can be completed in two basic actions: on the road transport took off at any time; in the sky, land transport at any time.

\subsubsection{Under the Sea}

For coastal cities, if you travel in no hurry to reach their destination, so people can choose to travel into the sea, so that vehicles on the road the number be reduced; same time, the marine traffic or leisure, entertainment, surfing is a good project. New offer of transportation to the public a wide range of travel, highlighting the human oriented thought, happy in the realization of the people in traffic. For example, in 2008 the Swiss car design company Rin-speed (Cloud-speed) launched the world's first concept car sQuba [5]. The car can be as common as cars on the highway; also be the same as the submarine or underwater vehicle in the water, very human.

Traffic congestion in the road, the entrance through the set, part of the transport moved to Airbus; by landing port, transport, transfer to another part of the sky; through the next to the sea, there are still some vehicles transferred to the ocean. Through the above three methods, the physical segregation of the three types of transport, the congestion in the number of road transport will be greatly reduced, along with traffic congestion problems 
solved.

\subsection{To Solve the Two Questions}

\subsubsection{Transfer One-Way Loop Road is Not Congested?}

This understanding may be affected by a single line, to explain the one-way loop road is not exactly the same one-way street. Establishment of one-way loop road network is to increase the number of (multi) "air highway" or "sea highway", the transport means to the limit, a combination to achieve the sharing of resources, improve transportation efficiency To further improve the traffic network system. As part of the car to release the single loop road to the sky or the sea, thus reducing the number of ground transportation, it is to solve the traffic congestion rather than moving plug points.

\subsubsection{Aircraft Will Not Happen between the Collision?}

This fear is very necessary and must be addressed. Cellular transport network in each individual ring, the aircraft are all one-way flight, aircraft, completed only in the node to switch between different regions, so the aircraft does not occur relative collision phenomenon, and, as the field of urban space further open space can also be layered to set Road, greatly enriched and facilitated the free navigation of aircraft.

\subsection{To Resolve a Contradiction}

New traffic plan, must reach the old city construction and traffic management, innovation and old-fashioned dispute exists, the formation of a contradiction. New traffic plan may also span to multiple independent administrative, and even across countries. On the one hand cellular network planning to follow the traditional rules of the game. Cultural monuments such as experience, military areas and other special circumstances, in order to build a large circle, put a small circle. On the other hand strict implementation of traffic planning. Within the red line in the planning of the "obstacles" to vigorously demolition, involving land, compensation and other specific issues, through political, economic and other means to a reasonable solution; encountered transnational transport planning, to use diplomatic means to give up the struggle, the traffic between the countries unite to achieve a planning multi-country pass.

With the progress of science and technology widely used in the transportation field, the unit already has a variety of vehicles travel. Urban transport planning and forward-looking cities to solve traffic congestion as one of important factors, transport planning is necessary to meet the current mode of transportation, but also consider the future direction of development of transport.

\section{Second, the Custom Software System to Solve Urban Congestion}

Establish a set of intelligent network traffic management software system, which is the living soul of cellular transport network is an important means to ensure the smooth flow of traffic. To enter the transit network to be free share for each vehicle traffic information, video and voice applications, pictures and understand and observe the network in a timely manner, the dynamic traffic conditions, and enhance the transparency of network operations. For example, the transportation network in place somewhere in a traffic accident or traffic jam, the computer network system immediately issued a directive, on the one hand to the other drivers to adjust the driving line, the other party inform traffic management officers in time, to resolve the problem, restore the traffic network. In addition, each state must adopt laws and ethics, and many other acts to regulate traffic, improve traffic efficiency and reduce the number of trips, reduce urban traffic pressure to ensure the smooth flow of large cities.

Traffic congestion in big cities in the world, many countries are facing a new task, but also a new problem. Rapid development in the international transport of the times, breaking the national boundaries, brought together national intelligence, share new results of traffic, the large flow of traffic for the world to do their own contribution.

\section{References}

[1] Proceedings of International Conference of Transportation Technology Innovation and Application of International Transport Infrastructure Construction and Maintenance Technology Conference, China Communications Press, April 2008, pp. 558-561.

[2] CCTV, "Beijing City Master Plan,” 2004. http://www. cctv.com/news/china/20040309/102266.shtml

[3] China Network Television, "New York: Make a Fly to Work the Air Train to Achieve Speed,” 2010. http://news. cntv.cn/world/20101128/104271.shtml

[4] People's Daily, "Days of the Guangzhou Metro was Forced to Burst, Urged the Public to Avoid It,” 2010. http://gd.people.com.cn/GB/123937/123964/1313828 8.html

[5] China Economic Net, "Car to be God into the Water Coming out High-Tech Alternative Models,” 2009. http://auto.ce.cn/main/wzqc/cb/tc/200902/13/t20090213_1819272 2.shtml 